\title{
Ultrasonografía transvaginal e histerosonografía en el diagnóstico de patologías endometriales
}

\author{
Ronald Salazar-Mora, ${ }^{1}$ Alberto Calderón-Zúñiga. ${ }^{2}$
}

Justificación y Objetivo: A pesar que en los últimos años la ultrasonografía transvaginal se ha convertido en una gran herramienta en la evaluación de pacientes con sangrado uterino anormal, ha mostrado cierta limitación en el diagnóstico de patología endometrial. La infusión salina intracavitaria en el momento de la realización de la sonografía ha demostrado ser un método más preciso. En el siguiente trabajo el objetivo principal fue comparar la sensibilidad y especificidad del ultrasonido transvaginal versus el uso de la histerosonografia en el diagnóstico de estas patologías.

Metodología: Treinta y seis pacientes con sangrado uterino anormal fueron seleccionadas de forma consecutiva, según referencia para ultrasonografía vaginal. A todas se les realizó un ultrasonido transvaginal y luego la histerosonografía, siendo los resultados comparados con el diagnóstico histológico, el cual fue considerado como el "estándar dorado".

Resultados: $38 \%$ de las pacientes mostraron un endometrio normal, el 30\% tuvo pólipo endometrial, 30\% tuvo hiperplasia endometrial, $19 \%$ miomas intramurales y/o adenomiosis y $7.6 \%$ miomas submucosos. La sensibilidad y especificidad del ultrasonido transvaginal fue de un $42.8 \%$, y $91.6 \%$ respectivamente, comparada con de $92.8 \%$ y $100 \%$ para la histerosonografía.

Conclusiones: La histerosonografía es más precisa en el diagnóstico de patología endometrial en pacientes con sangrado uterino anormal.

Descriptores: sonohisterografía, histerosonografía, endometrio, ultrasonido transvaginal, pólipos endometriales, miomas intrauterinos, hiperplasia endometrial, postmenopausia

Recibido: 7 de marzo de 2001.

Aceptado: 10 de octubre de 2001.

\section{Introducción}

La infusión de solución salina dentro de la cavidad endometrial durante la realización de la ultrasonografía transvaginal mejora la imagen y el diagnóstico de las enfermedades endometriales. A esta técnica se le denomina histerosonografía. ${ }^{1}$

En 1981 Nannini y col${ }^{2}$ fueron los primeros en describir esta técnica. En 1986, Randolph et al, ${ }^{3}$ usando solución salina

\footnotetext{
Abreviaturas: ISI, infusión salina intracavitaria; THR, tratamiento hormonal de reemplazo.

1 Residente Postgrado de Ginecología y Obstetricia, Hospital de las Mujeres "Adolfo Carit Eva".

2 Clínica Servicio de Ginecología, Hospital de las Mujeres "Adolfo Carit Eva".
}

Correspondencia: Ronald Salazar Mora, dirección: Heredia 250mts oeste de la estación de Bomberos, teléfono 391-12-86, fax 261-12-42. E mail: ronitol@latinmail.com intrauterina en combinación con la ultrasonografía abdominal, identificaron correctamente 53 anormalidades en 54 pacientes que luego fueron sometidas a histerectomía.

En 1993, Parsons y Lense ${ }^{4}$ modificaron la técnica utilizando la ultrasonografía transvaginal. En este estudio fueron evaluadas 39 pacientes por sangrado uterino anormal 16 tenían pólipos endometriales, 4 hiperplasia endometrial, 2 cáncer de endometrio, 2 endometrio normal y todos los diagnósticos fueron confirmados histológicamente.

Goldstein et $a l^{5}$ reportaron los beneficios de la infusión salina intracavitaria (ISI) en pacientes perimenopáusicas con sangrado. Los autores reconocieron las limitaciones de la ultrasonografía transvaginal en la evaluación del endometrio en estas pacientes.

Basados en los resultados de estos investigadores nos planteamos el objetivo del presente estudio con el fin de determinar la sensibilidad y especificidad de la histerosonografía, comparada con la ultrasonografia transvaginal de alta 
resolución para el diagnóstico de enfermedades endometriales, tomando como estándar de oro el reporte histológico.

\section{Materiales y Métodos}

Se trata de un estudio prospectivo que va de 01 marzo a 31 Julio del 2000, en el se incluyeron en forma consecutiva un total de 36 mujeres con diagnóstico de sangrado uterino anormal, ellas fueron referidas para estudio sonográfico y cumplían los criterios de inclusión establecidos para este estudio diagnóstico.

La edad promedio de las pacientes fue de 42 años. A ellas se les realizó un ultrasonido transvaginal y luego se practicó la infusión de suero salino intracavitario (histerosonografía). Ambos resultados presuntivos se corroboraron luego con el reporte histológico, (biopsia de endometrio, legrado instrumental o histerectomía), y de esa forma se calculó la sensibilidad y especificidad de cada uno de los métodos.

Técnica de la histerosonografía: El procedimento se realizó después de obtener el consentimiento informado de la paciente. Se insertó el espéculo a la paciente visualizando el cervix, el cual se limpia con solución de yodo. Se introdujo entonces en la cavidad una sonda nasogástrica de niño \#8 o una sonda foley \#8 en aquellas cervices muy dilatados que pudieran permitir el escape del fluido. Antes de introducir el cateter, éste fue "purgado" para evitar que las burbujas de aire produzcan un artefacto dentro de la cavidad. Una vez introducido el catéter se retiró el espéculo estéril y se introdujo el transductor vaginal del ultrasonido, se inició entonces la infusión de 20 a 40 cc de solución salina a través del catéter y se visualizó en tiempo real la separación del endometrio (Figura 1). Se realizaron barridos de cuerno a cuerno (transversales) y longitudinales, midiendo por separado el grosor del endometrio (cara anterior y posterior). Ambos procedimientos fueron realizados en 10 a 15 minutos. No se reportaron complicaciones mayores ni menores.

\section{Resultados}

En la mayoría de las pacientes se encontró un endometrio normal, luego en orden de frecuencia aparecieron pólipos endometriales, hiperplasia endometrial y miomas submucosos. Ante la presencia de miomas, la histerosonografía permitió delimitar mejor su localización exacta y el tamaño (Cuadro 1).

La limitación del ultrasonido transvaginal en relación con la histerosonografía, se nota en el número de falsos negativos. Donde por el ultrasonido transvaginal se sospechaba la presencia de enfermedad, la histerosonografía y el resultado histológico lo descartaron (Cuadro 2).

\section{Discusión}

Al comparar nuestro resultados con la experiencia de otros autores, vemos el gran beneficio de esta modalidad para el diagnóstico más preciso de patología endometrial en pacientes

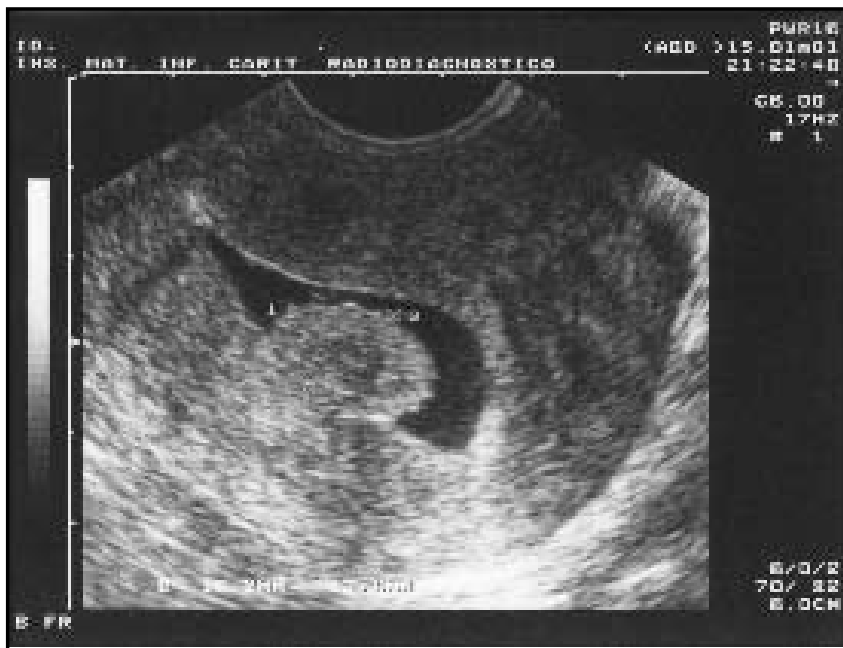

Figura 1: Paciente menopáusica de 52 años con THR, historia de sangrado uterino anormal de 1 año de evolución, reporte de ultrasonido convencional endometrio de $8 \mathrm{~mm}$ normal, se realiza histerosonografía y se visualiza pólipo endo metrial de $18 \times 12.8 \mathrm{~mm}$, con un endometrio atrofico < de $3 \mathrm{~mm}$ suma de cara anterior y posterior. La paciente se somete a cirugía histeroscópica resecando el pólipo y confirmando su diagnóstico.

\begin{tabular}{|c|c|}
\hline $\begin{array}{l}\text { Cuadro 1: Distribución porcentual } \\
\text { causas de sangrado uterino anorma } \\
\text { reporte } \\
\begin{array}{r}\text { histológico (biopsia, Leg } \\
\text { histerectomía) }\end{array}\end{array}$ & $\begin{array}{l}\text { de las } \\
\text { I según } \\
\text { ado, pieza }\end{array}$ \\
\hline Causa de sangrado uterino anormal & Frecuencia \\
\hline Endometrio normal: proliferativo o secretor, etc. & $38 \%$ \\
\hline Pólipo endometrial & $30 \%$ \\
\hline Hiperplasia endometrial(incluye simple y compleja) & $23 \%$ \\
\hline Adenomiosis, miomas intramurales & $19 \%$ \\
\hline Miomas submucosos & $7.6 \%$ \\
\hline
\end{tabular}

con sagrado uterino anormal, siendo los resultados obtenidos similares a los reportados por la literatura revisada.

Durante la realización de la histerosonografia el endometrio normal aparece simétrico y bien demarcado, por lo que se pueden medir ambas capas por separado. En la premenopausia el grosor va de 4 a $8 \mathrm{~mm}$ durante la fase proliferativa, y de 8 a 14 $\mathrm{mm}$ durante fase secretora, 6 mientras que en la paciente posmenopáusica es anormal todo endometrio con grosor mayor a $5 \mathrm{~mm}$ y ello exige un estudio histológico. La histerosonografía permite seleccionar con gran exactitud cuáles de estas pacientes requieren o no estudio histológico.

Kupfer et $a l,{ }^{7}$ encontraron que el pólipo endometrial engruesa el endometrio y crea una imagen de hiperplasia endometrial en el ultrasonido transvaginal, sin embargo en la histerosonografía se logra visualizar, y ubicar el pólipo en un 100\% de los 


\section{Cuadro 2: Comparación entre el diagnóstico presuntivo de las dos pruebas y el resultado histológico}

\begin{tabular}{lcc}
\hline Parámetros & $\begin{array}{l}\text { Resultado } \\
\text { Ultrasonido } \\
\text { Transvaginal }\end{array}$ & $\begin{array}{l}\text { Resultado } \\
\text { Histerosonografía }\end{array}$ \\
\hline Verdaderos positivos & 6 & 13 \\
Verdaderos negativos & 11 & 12 \\
Falsos positivos & 1 & 0 \\
Falsos negativos & 8 & 1 \\
Sensibilidad & $42.8 \%$ & $92.8 \%$ \\
Especificidad & $91.6 \%$ & $100.0 \%$ \\
VPPP & $85.7 \%$ & $100.0 \%$ \\
VPPN & $57.8 \%$ & $92.3 \%$ \\
\hline Para una $\mathrm{n}=36$ pacientes & & \\
\hline
\end{tabular}

casos, y brinda información de si su base es sésil o pediculado, lo cual es de gran ayuda para decidir el tipo de cirugía a practicar (legrado a ciegas, resección histeroscópica, etc).

Además, el pólipo y el mioma submucosos tienen características sonográficas particulares en la histerosonografía.

Los miomas muchas veces son difíciles de localizar con exactitud con la ultrasonografía convencional. La histerosonografía tiene grandes ventajas ya que ubica el mioma y lo clasifica de acuerdo a su localización, tamaño y grado de extensión intramural. ${ }^{8}$ De esa forma se puede valorar la resectabilidad histeroscópica y ser menos agresivos en el tratamiento de muchas pacientes.

Todas las anteriores experiencias, comparadas con las de otros investigadores, se corroboraron en nuestro estudio, tal y como lo demuestran los resultados obtenidos. El gran beneficio de la histerosonografía en pacientes con sangrado uterino anormal en edad reproductiva, es el obtener mayor certeza diagnóstica de las patologías endometriales y la localización de estas lesiones como muestran nuestros resultados. En la mujer postmenopáusica con sangrado las ventajas son mayores y actualmente, al igual que nosotros, muchos autores la recomiendan, como examen inicial en la valoración del sangrado en este grupo de pacientes, ${ }^{9}$ para luego obtener el especímen histológico con mayor precisión diagnóstica.

\section{Abstract}

Justification and objectives: In spite than in the last years the vaginal ultrasound has become a powerful tool in the evaluation of patients with abnormal uterine bleeding, it has shown some limitations for the diagnosis of endometrial pathology.

Intracavitary saline infusion during the ultrasonographic procedure, has shown to be a more sensitive method.

Our main objective was to compare the sensitivity and specificity of the vaginal ultrasound versus the histerosonography in the diagnosis of various endometrial pathologies.

Methodology: In this study, 36 patients with the diagnosis of abnormal uterine bleeding were selected consecutively as they were refered for vaginal ultrasound.

All of them had vaginal ultrasound and then histerosonography, comparing the results with the histologic diagnosis as the " gold stándar".

Results: Thirty eight percent had normal endometrium 30\% had endometrial polyp, 30\% had endometrial hyperplasia, $19 \%$ intramural myomas and/or adenomyosis, and submucus myomas were found in $7.6 \%$ of the patients.

The sensitivity and specificity for the vaginal ultrasound was $42.8 \%$ and $91.6 \%$ and for the histerosonography were $92.8 \%$ y $100 \%$ respectively.

We conclude that histerosonography was more precise in the diagnosis of endometrial pathology in patients with abnormal uterine bleeding.

\section{Agradecimiento}

Queremos agradecer al Servicio de Radiología del Hospital de las Mujeres la ayuda brindada para la realización de esta investigación.

\section{Referencias}

1. Cullinan JA, Fleischer AC, Kepple DM, et al: Sonohysterography: A techninque for endometrial evaluation. Radigraphics 1995; 15: 501514.

2. Nannini R, Chelo E, Branconi F, et al: Dynamic echohysteroscopy: A new diagnostic technique in the study of female infertility. Acta Eur Fertil 1981; 12: 165-171.

3. Randolph JR, Ying YK, Maier DB, et al. Comparison of real-time ultrasonography, hysterosalpingraphy, and laparoscopy/hysteroscopy in evaluation of uterine abnormalities and tubal patency. Fertil Steril 1986; 46: 828-832.

4. Parsons AK, Lense JJ. Sonography for endometrial abnormalities: Preliminary results. J Clin Ultrasound 1993; 231: 87-95.

5. Goldstein SR, Zeltser I, Horan CK, et al. Ultrasonography-based triage for perimenopausal patiens with abnormal uterine bleeding. Am J Obstet Gynecol 1997; 177: 102-108.

6. Granberg S, Wikland M, Karlsson B, et al. Endometrial thickness as measured by endovaginal ultrasonography for identifying endometrial abnormality. Am J Obstet Gynecol 1991; 64: 47-52.

7. Kupfer M, Schiller V, Hansen G, et al. Transvaginal sonographic evaluation of endometrial polyps. J Ultrasound Med 1994; 13: 535-539.

8. Shimizu B, Fukuda K, Yomura W, et al. Transvaginal hysterosonography for differential diagnosis between submucous and intramural myoma. Gynecol Obstet Invest 1993; 35: 236-239.

9. Cacciatore B, Ramsay T, Lehtovirta P, et al. Transvaginal sonography and hysteroscopy in postmenopausal bleeding. Acta obstet Gynecol Scand 1994; 73: 413-416. 